\title{
RESEARCH
}

Open Access

\section{Refining sleep measurement using the Motionwatch8@: how many days of monitoring do we need to get reliable estimates of sleep quality for older adults with mild cognitive impairment?}

\author{
Ryan S. Falck ${ }^{1,2,3}$, Cindy K. Barha ${ }^{1,2,3}$, Patrick C. Y. Chan ${ }^{1,2,3}$ and Teresa Liu-Ambrose 1,2,3* $^{\text {(1) }}$
}

\begin{abstract}
Background: Poor sleep is common among older adults with mild cognitive impairment (MCl)—a transition stage between healthy cognition and dementia. Objective, reliable, and low-burden field methods to measure older adult sleep are also currently needed. The MotionWatch8@ (MW8) wrist-worn actigraph provides estimates of sleep with 14 days of observation; however, there may be underlying differences in the reliability of sleep estimates based on $\mathrm{MCl}$ status. We therefore investigated the number of MW8 monitoring days required to estimate sleep in older adults with $\mathrm{MCl}$ and without.
\end{abstract}

Methods: Older adults ( $55+$ years; $N=151$ ) wore the MW8 for $\geq 14$ days. The Montreal Cognitive Assessment was used to categorize participants with probable $\mathrm{MCl}$ (scores of <26/30) and participants without $\mathrm{MCl}(\geq 26 / 30)$. We calculated intra-class reliability coefficients for one, seven, and 14 days of wear-time, and performed SpearmanBrown predictions to determine the number of monitoring days needed for an ICC $=0.80$.

Results: Older adults with $\mathrm{MCI}$ were older $(p<0.01)$, more likely to be male $(p=0.03)$, and had shorter sleep duration $(p<0.01)$. Spearman-Brown analyses indicated that the number of monitoring days needed for an ICC $=$ 0.80 in older adults with probable $\mathrm{MCl}$ was 7 days for sleep duration, 4 days for fragmentation, and 4 days for efficiency; adults without $\mathrm{MCl}$ required 4 days for duration, 6 days for fragmentation, and 3 days for efficiency. Conclusions: Our results indicate that while the reliability of MW8 estimates of sleep differs based on cognitive status, 7 days of MW8 monitoring provides reliable estimates of sleep for adults with $\mathrm{MCl}$ and those without. Keywords: Sleep, Actigraphy, Measurement, Reliability, Older adults, Mild cognitive impairment

\footnotetext{
* Correspondence: teresa.ambrose@ubc.ca

'Aging, Mobility and Cognitive Neuroscience Laboratory, Department of Physical Therapy, Faculty of Medicine, University of British Columbia,

212-2177 Wesbrook Mall, Vancouver, BC V6T 1Z3, Canada

2Djavad Mowafaghian Centre for Brain Health, University of British Columbia,

Vancouver, BC, Canada

Full list of author information is available at the end of the article
}

(c) The Author(s). 2020 Open Access This article is licensed under a Creative Commons Attribution 4.0 International License, which permits use, sharing, adaptation, distribution and reproduction in any medium or format, as long as you give appropriate credit to the original author(s) and the source, provide a link to the Creative Commons licence, and indicate if changes were made. The images or other third party material in this article are included in the article's Creative Commons licence, unless indicated otherwise in a credit line to the material. If material is not included in the article's Creative Commons licence and your intended use is not permitted by statutory regulation or exceeds the permitted use, you will need to obtain permission directly from the copyright holder. To view a copy of this licence, visit http://creativecommons.org/licenses/by/4.0/ 


\section{Background}

Promoting older adult cognitive health is a public health priority since the number of older adults with cognitive impairment and dementia is increasing (Jacqmin-Gadda et al., 2013). Lifestyle and behavioural modification strategies which promote or maintain cognitive health are thus a frontline, low-cost, and increasingly popular line of research inquiry.

Good quality sleep is one lifestyle factor which is a pillar of healthy cognitive aging (Scullin, 2017; Yaffe et al., 2014; Liu-Ambrose \& Falck, 2019). Sleep is critical for the maintenance of cognitive function and for brain function (Lowe et al., 2017; Banks \& Dinges, 2007; Killgore, 2010). While the importance of sleep for cognitive health does not change with age (Scullin, 2017), there are changes in sleep which occur as a normal part of the aging process - both in terms of decreased quality and quantity (Crowley, 2011; Espiritu, 2008). However, more than half of adults over 65 years of age report at least one chronic sleep complaintthe most common being the inability to stay asleep at night (Foley et al., 1995). One reason is age-associated changes in the sleep-wake cycle of older adults (Crowley, 2011; Landry \& Liu-Ambrose, 2014). For example, homeostatic drive (i.e., Process S) declines with age (Borbély et al., 2016). Older adults also commonly report excessive daytime sleepiness-which is a key indicator of accumulated sleep debt (Espiritu, 2008).

While changes in sleep thus appear to be an unavoidable consequence of aging (Scullin, 2017; Yaffe et al., 2014; Crowley, 2011), older adults who sleep poorly are also at increased risk for mild cognitive impairment (MCI) and dementia (da Silva, 2015). MCI is classified as cognitive decline greater than expected for age and education level which does not interfere with independence (Petersen et al., 1999). An estimated $10-20 \%$ of adults over 65 years of age are living with MCI (Langa \& Levine, 2014), which is associated with up to a $30 \%$ increased risk of developing dementia within 5 years (Petersen, 2004). MCI thus represents a transition period between healthy cognitive aging and dementia, wherein lifestyle factors such as sleep may be critical to maintaining cognitive health (Petersen et al., 2001). Importantly, older adults with $\mathrm{MCI}$ are more likely to experience poor sleep than healthy older adults (Naismith et al., 2014), and poor sleep is associated with an increased risk of progression from MCI to dementia (Tranah et al., 2011). Animal models indicate that chronic poor sleep leads to increased cortical amyloid-beta $(\mathrm{A} \beta)-\mathrm{a}$ hallmark pathology of Alzheimer's disease (Roh et al., 2012). Rodents which are deprived of sleep experience increased A $\beta$ production (Kang et al., 2009), while in vivo animal models suggest sleep promotes the clearance of A $\beta$ (Xie et al., 2013). Sleep is thus a critical pathway by which the brain appears to maintain cognitive health
(Scullin, 2017; Yaffe et al., 2014; Liu-Ambrose \& Falck, 2019). When this pathway is disrupted, a vicious cycle of accelerating cognitive decline may occur-whereby poor sleep may lead to increasing cognitive decline, and viceversa (Ju et al., 2013).

Valid and reliable field methods for measuring sleep are thus needed to understand the impact of sleep on cognitive health. Sleep can be measured objectively using polysomnography, or it can be estimated using wristworn and hip-worn actigraphy (Krystal \& Edinger, 2008). In addition, sleep can be estimated subjectively by questionnaire (Zinkhan et al., 2014; Landry et al., 2015a). The gold standard for measuring sleep is polysomnography (Scullin \& Bliwise, 2015); however the invasive nature of polysomnography-usually requiring an overnight stay in a sleep laboratory or clinic-makes long-term multi-night recordings impractical. Subjective measures of sleep are quick and easy to administer and score, and can discriminate "good" vs. "poor" sleepers, but they are not able to detect subtle but clinically important changes in sleep due to age or disease (Landry et al., 2015a). Estimating sleep using actigraphy is therefore an increasingly popular alternative for measuring sleep, although it is open to issues of validity and reliability compared to polysomnography (Scullin \& Bliwise, 2015). Nonetheless, actigraphy does provide an objective method of estimating sleep in a natural environment which can span multiple nights, thus delivering a clearer illustration of an older adult's usual sleep pattern.

One readily-available actigraph capable of measuring sleep quality is the Motionwatch8๑ wrist-worn accelerometer (MW8; (Landry et al., 2015b)). Previous investigations with the device have observed sleep quality over a period of 14 days (Landry et al., 2015a; Falck et al., 2018), since this measurement protocol is based on current guidelines and past analyses of actigraphy (Ancoli-Israel et al., 2015; Van Someren, 2007); however, reducing the number of days necessary to wear the MW8 could reduce participant burden. Moreover, since older adults with MCI appear to have different sleep than their healthy cognitive counterparts (Naismith et al., 2014; Tranah et al., 2011), it is plausible that the MW8 may have different estimates of reliability for the measurement of sleep based on cognitive status. Hence, we examined the necessary number of days for obtaining reliable estimates of sleep in older adults with $\mathrm{MCI}$ and those without.

\section{Methods}

Ethical approval for this study was obtained from the Vancouver Coastal Health Research Institute and the University of British Columbia's Clinical Research Ethics Board (H14-01301). All participants provided written informed consent. 


\section{Participants}

Data for this study were collected as part of the Sleep and Cognition Study, a cross-sectional study examining the associations between sleep quality and cognition among older adults (Falck et al., 2017). We recruited and collected data between August 27, 2014 and June 30, 2016. Details of the full study protocol can be found elsewhere (Falck et al., 2017). We recruited 153 older adults from Vancouver, British Columbia by advertisements placed in local community centres, newspapers, and word of mouth referrals. Interested individuals were initially pre-screened for eligibility criteria.

Participants were included if they: 1) were 55+ years of age living the Greater Vancouver area; 2) scored $\geq 24 / 30$ on the Mini-Mental State Examination (MMSE), with scoring of attention being performed using serial sevens (Folstein et al., 1975); and 3) were able to read, write, and speak English with acceptable visual and auditory acuity. Participants were excluded if they were: 1) diagnosed with dementia of any type; 2) diagnosed with another type of neurodegenerative or neurological condition; 3) taking medications that may negatively affect cognitive function; 4) planning to participate or were currently enrolled in a clinical drug trial; or 5) unable to speak as judged by an inability to communicate by phone. Individuals were not excluded based on use of medications which may affect sleep quality (either negatively or positively), nor were participants selected on the basis of their sleep.

\section{Study design and measurement}

At study entry, we ascertained general health, subjective sleep quality using the Pittsburgh Sleep Quality Index (PSQI; (Buysse et al., 1989)), height to the nearest $0.1 \mathrm{~cm}$ using a stadiometer, weight to the nearest $0.1 \mathrm{~kg}$ using an electronic scale, demographics, socioeconomic status, and education by a questionnaire. Height and weight were used to calculate body mass index (BMI; $\mathrm{kg} / \mathrm{m}^{2}$; (Garrouste-Orgeas et al., 2004)). Global cognitive function was assessed by the MMSE (Folstein et al., 1975), and the Montreal Cognitive Assessment (MoCA; (Nasreddine et al., 2005)). We categorized participants based on MCI status with a score of $<26 / 30$ on the MoCA indicating probable MCI, which has been found to have good internal consistency and test-retest reliability, and was able to correctly identify $90 \%$ of a large sample of $\mathrm{MCI}$ individuals from two different clinics (Nasreddine et al., 2005).

Participants were then fitted with the MW8 and provided detailed information on its features (i.e., the light sensor, event marker button, and status indicator). Participants were instructed to press the event marker button each night when they started trying to sleep, and again each morning when they finished trying to sleep. Consistent with established protocol for wrist-worn actigraphy, participants wore the MW8 on the nondominant wrist (Ancoli-Israel et al., 2015; Sadeh, 2011).

Participants were also given the 9-item Consensus Sleep Diary (CSD) and asked to complete it each morning upon waking (Carney et al., 2012). The responses from the CSD were used to confirm sleep windows as determined by the time stamped event markers. In cases where the event marker and CSD entry disagreed for the start time of the sleep window, we used activity cessation and light sensor data from the MW8 to determine "lights out". Similarly, when the event marker and CSD entry disagreed for the end of the sleep window, we used activity onset and "lights on" to determine the end of the sleep window. If responses from the CSD entry disagreed with the event markers entered by participants as the start of the day (i.e., participant finished trying to sleep, and was awake and out of bed), we used activity onset and light sensor data to determine the start of the day. Similarly, when the event marker and CSD entry disagreed for the end of day (i.e., participant in bed and trying to sleep), we used activity cessation and light sensor data to determine the end of the day. In accordance with the currently established protocol for measuring sleep quality via actigraphy (Ancoli-Israel et al., 2015; Sadeh, 2011), each participant was continuously monitored for a minimum of 14 nights. After collection, stored activity counts were downloaded and saved to an IBM compatible computer for subsequent data reduction and analysis.

\section{MW8 instrumentation and data reduction}

We measured sleep using the MW8 actigraphy system (CamNtech; Cambridge, United Kingdom). The MW8 is a tri-axial accelerometer designed to observe acceleration ranging in magnitude from $0.01 \mathrm{G}$ to $8 \mathrm{G}$, with a frequency of $3-11 \mathrm{~Hz}$. The filtered acceleration signal is digitized and the magnitude is summed over a user-specified time interval. At the end of each interval, the summed value or activity "count" is stored in memory and the integrator is reset. The MW8 is the updated version of the Actiwatch7, an actigraph with evidence of validity against polysomnography in healthy adults (Mean age: $30 \pm 6$ years; $45 \%$ female; (O'Hare et al., 2015)), and also adults with chronic insomnia (Mean age: $41 \pm 12$ years; $78 \%$ female; (Martoni et al., 2012)). There is also initial evidence of validity against polysomnography for the MW8 among 1) 54 adults with suspected sleep disorders including obstructive sleep apnea, insomnia, hypersomnia, and Ehlers Danlos syndrome (Mean Age: $53 \pm 16$ years; 61\% female); and 2) 19 healthy adults (Mean Age: $28 \pm 5$ years; $53 \%$ female; (Elbaz et al., 2012)). For the current study, we used $60 \mathrm{~s}$ epochs (Ancoli-Israel et al., 2015; Sadeh, 2011).

Data were analyzed using MotionWare 1.0.27 (camntech) to estimate different sleep indices including: fragmentation index, sleep efficiency (time asleep expressed 
as a percentage of time in bed), sleep duration (total time spent sleeping), sleep latency (time between "lights out" and falling asleep), and wake after sleep onset (time spent awake after sleep has been initiated and before final awakening). Fragmentation index is a description of restlessness while sleeping and is defined by MotionWare as the sum of 1) the total time spent sleeping categorized as mobile in the epoch-by-epoch mobile/ immobile categorization expressed as a percentage of the time spent asleep; and 2) the number of immobile bouts which were equal to $1 \mathrm{~min}$ in length expressed as a percentage of the total number of immobile bouts during time spent sleeping. Only minutes categorized as asleep were included in the calculation of fragmentation index.

\section{Statistical analyses}

We performed all of our statistical analyses using $\mathrm{R}$ version 3.3.1 using the psych, Hmisc, and ICC packages. Our statistical code can be found in Supplementary Material S1. Two participants did not complete the MoCA. These individuals were removed from analyses such that our final sample was 151 participants.

\section{Participant characteristics based on probable $\mathrm{MCl}$ status}

We calculated means and standard deviations for all variables of interest based upon probable MCI status (i.e., MoCA score $<26 / 30$ ). We determined demographic differences in probable MCI status using independent sample $t$-tests for continuous variables and chi-square tests for categorical variables, using probable MCI status (yes/ no) as the grouping variable. Subsequently, we performed analyses of covariance (ANCOVA) to determine differences in estimates of sleep quality based on probable MCI status. We performed separate ANCOVA models for each of our measures estimating sleep quality (i.e., PSQI total score, PSQI sleep duration, and MW8 measured sleep duration, fragmentation index, sleep efficiency, sleep latency, and wake after sleep onset). Within each ANCOVA model, we controlled for age, sex, and sleep medication use while using probable MCI status as the grouping variable.

\section{Reliability of the MW8 for estimating sleep quality based on probable $\mathrm{MCl}$ status}

We then calculated between-day intraclass correlations (ICC) and 95\% confidence intervals (CIs) for one, four, seven and 14 days of monitoring, and classified ICCs according to the criteria of Koo and Li (Koo \& Li, 2016). For single day ICC's we used a single absolute intraclass correlation coefficient $\left(\mathrm{ICC}_{1,1}\right)$ to determine single day expected reliability using the following formula (Denegar \& Ball, 1993):

$$
\frac{B M S-E M S}{B M S(k-1) E M S+k\left(\frac{T M S-E M S}{N}\right)}
$$

Wherein, $B M S$ is the between subject mean square, the EMS is the residual error, TMS is the trial mean square, $k$ refers to the number of trials (in this case, one trial), and $N$ is the number of participants. We used all 14 days of data to calculate our single day ICCs. For our analysis of multiple day reliability, we used average random raters $\left(\mathrm{ICC}_{2, \mathrm{k}}\right)$ using the same formula, wherein $k$ was the number of days monitored. For our calculations of four and 7 day reliability, we only used data from the first 4 and 7 days, respectively.

We also calculated the required days of monitoring needed to achieve ICC's of $0.70,0.80$ and 0.90 using the Spearman-Brown prophecy formula (Hopkins, 1998). Subsequently, we calculated separate ICCs and Spearman-Brown prophecies for 1) participants with probable $\mathrm{MCI}$ and 2) those without MCI. We then performed $z$ tests to determine if ICC estimates for our different sleep parameters differed significantly by MCI status after one, four, seven, and 14 days of monitoring.

\section{Results}

Participant characteristics based on probable $\mathrm{MCl}$ status Participant characteristics are described in Table 1 . Mean participant age was 71.19 years $(\mathrm{SD}=7.26$; Range: $55-101$ years), $66.89 \%$ of the sample was female, and $77.48 \%$ were retired. Average BMI was $26.71 \mathrm{~kg} / \mathrm{m}^{2}$ $\left(\mathrm{SD}=5.05\right.$; Range: $\left.17.08-42.40 \mathrm{~kg} / \mathrm{m}^{2}\right), 13.25 \%$ of the sample used sleep medications, and average MMSE score was 28.89 ( $\mathrm{SD}=1.11$; Range: 25-30). Average PSQI score was 7.28 ( $\mathrm{SD}=4.00$; Range: $1-18)$, and participants reported sleeping an average of $373.60 \mathrm{~min} /$ day (SD = 73.43; Range: 120-570 min/day) on the PSQI. Participants had an average MW8 measured sleep duration of $401.10 \mathrm{~min} /$ day $(\mathrm{SD}=51.23$; Range: $164.85-541.77$ $\mathrm{min} /$ day), average fragmentation index of 31.17 ( $\mathrm{SD}=$ 11.03; Range: 11.21-57.15), and average sleep efficiency of $82.57 \%$ ( $\mathrm{SD}=6.10$; Range: $66.74-94.99 \%$ ).

Older adults with probable MCI were significantly older $(p=0.01)$ and had poorer performance on the MMSE $(p<0.01)$. The proportion of males categorized as MCI was greater than the proportion of males categorized as cognitively healthy $(p=0.03)$. Older adults with probable MCI had significantly shorter subjective sleep duration on the PSQI $(p=0.01)$ and shorter MW8 measured sleep duration $(p<0.01)$ than their healthy cognitive peers after controlling for age, sex, and sleep medication use. 
Table 1 Participant Characteristics Mean (SD) or \%

\begin{tabular}{|c|c|c|c|c|}
\hline Participant Characteristic & $\begin{array}{l}\text { All } \\
\text { Participants } \\
(N=151)\end{array}$ & $\begin{array}{l}\text { Older Adults without Mild Cognitive Impairment ( } N= \\
\text { 69) }\end{array}$ & $\begin{array}{l}\text { Older Adults with Mild Cognitive } \\
\text { Impairment } \\
(N=82)\end{array}$ & $\begin{array}{l}p- \\
\text { value* }\end{array}$ \\
\hline Age & $71.19(7.26)$ & $69.42(6.36)$ & $72.67(7.66)$ & $<0.01$ \\
\hline$\%$ Female & $66.89 \%$ & $76.81 \%$ & $58.54 \%$ & 0.03 \\
\hline Body Mass Index $\left(\mathrm{kg} / \mathrm{m}^{2}\right)$ & $26.71(5.05)$ & $25.91(5.16)$ & $27.45(4.87)$ & 0.13 \\
\hline \%Retired & $77.48 \%$ & $78.26 \%$ & $76.83 \%$ & 0.99 \\
\hline \multicolumn{5}{|l|}{ Education } \\
\hline Less than high school diploma & $4.64 \%$ & $1.45 \%$ & $7.32 \%$ & \multirow[t]{5}{*}{0.26} \\
\hline High school diploma & $13.91 \%$ & $14.49 \%$ & $13.41 \%$ & \\
\hline Trade school & $11.26 \%$ & $7.25 \%$ & $14.63 \%$ & \\
\hline Some university & $15.89 \%$ & $14.49 \%$ & $17.07 \%$ & \\
\hline University degree or higher & $54.30 \%$ & $62.32 \%$ & $47.56 \%$ & \\
\hline \multicolumn{5}{|l|}{ Smoking History } \\
\hline Current Smoker & $1.32 \%$ & $1.45 \%$ & $1.22 \%$ & \multirow[t]{3}{*}{0.96} \\
\hline Past Smoker & $49.67 \%$ & $50.72 \%$ & $48.78 \%$ & \\
\hline Non-Smoker & $49.01 \%$ & $47.83 \%$ & $50.00 \%$ & \\
\hline \multicolumn{5}{|l|}{ Comorbidities } \\
\hline Hypertension & $26.49 \%$ & $17.39 \%$ & $34.15 \%$ & 0.03 \\
\hline Hypercholesterolemia & $17.22 \%$ & $13.04 \%$ & $20.73 \%$ & 0.30 \\
\hline Arthritis & $13.25 \%$ & $13.04 \%$ & $13.41 \%$ & 0.99 \\
\hline Mood Disorder & $7.95 \%$ & $10.14 \%$ & $6.10 \%$ & 0.54 \\
\hline Hypothyroidism & $15.89 \%$ & $20.23 \%$ & $12.20 \%$ & 0.26 \\
\hline Cardiovascular Disease & $16.56 \%$ & $7.25 \%$ & $24.39 \%$ & $<0.01$ \\
\hline Diabetes Mellitus & $2.65 \%$ & $1.45 \%$ & $3.66 \%$ & 0.74 \\
\hline Cancer & $1.99 \%$ & $0.00 \%$ & $3.66 \%$ & 0.31 \\
\hline Number of Medications & $2.27(2.15)$ & $2.01(1.75)$ & $2.49(2.43)$ & 0.17 \\
\hline Sleeping Medication Use & $13.25 \%$ & $17.39 \%$ & $9.76 \%$ & 0.26 \\
\hline $\begin{array}{l}\text { Diagnosed Obstructive Sleep } \\
\text { Apnea }\end{array}$ & $3.57 \%$ & $0.00 \%$ & $7.17 \%$ & 0.99 \\
\hline CPAPa Use & $3.57 \%$ & $0.00 \%$ & $7.17 \%$ & 0.99 \\
\hline MMSE ${ }^{\mathrm{b}}$ Score & $28.89(1.11)$ & $29.22(0.87)$ & $28.61(1.21)$ & $<0.01$ \\
\hline MoCAc Score & $24.79(2.83)$ & $27.19(1.10)$ & $22.77(2.19)$ & $<0.01$ \\
\hline \multicolumn{5}{|l|}{ Pittsburgh Sleep Quality Index Measures } \\
\hline Total Score & $7.28(4.00)$ & $6.62(5.44)^{*}$ & $7.47(4.71)^{*}$ & $0.15^{*}$ \\
\hline $\begin{array}{l}\text { Subjective Sleep Duration (min/ } \\
\text { day) }\end{array}$ & $373.60(73.43)$ & $393.89(111.30)^{*}$ & $363.23(96.99)^{*}$ & $0.01^{*}$ \\
\hline \multicolumn{5}{|l|}{ MotionWatch8 Sleep Measures } \\
\hline Sleep Duration (min/day) & $401.10(51.23)$ & $415.75(79.05)^{*}$ & $390.18(68.87)^{*}$ & $<0.01^{*}$ \\
\hline Fragmentation Index & $31.17(11.03)$ & $30.51(16.68)^{*}$ & $32.71(14.76)^{*}$ & $0.23^{*}$ \\
\hline Sleep Efficiency & $82.57(6.10)$ & $82.95(9.06)^{*}$ & $82.05(8.52)^{*}$ & $0.40^{*}$ \\
\hline Sleep Latency (min/day) & $6.72(9.00)$ & $6.14(13.70)^{*}$ & $7.35(11.88)^{*}$ & $0.41^{*}$ \\
\hline Wake After Sleep Onset (min/day) & $85.70(34.43)$ & $84.92(53.52)^{*}$ & $87.78(47.40)^{*}$ & $0.63^{*}$ \\
\hline Wake After Sleep Onset (min/day) & $85.70(34.43)$ & $84.92(53.52)^{*}$ & $87.78(47.40)^{*}$ & $0.63^{*}$ \\
\hline
\end{tabular}

* $P$-values refer to group differences (i.e., $\mathrm{t}$-tests, chi-square, or ANCOVA) between Older Adults with Mild Cognitive Impairment and those without ${ }^{*}$ Controlling for age, sex, sleep medication use

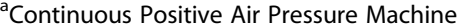

${ }^{\mathrm{b}}$ Mini Mental State Exam

cMontreal Cognitive Assessment 


\section{Reliability coefficients} All participants $(N=151)$

Intraclass reliability coefficients and 95\% CIs for mean daily activity levels derived from one, four, seven and 14 days are shown in Table 2 . The reliability coefficients exhibit a trend towards greater reliability for sleep duration, fragmentation index, sleep efficiency, and wake after sleep onset. Overall, there was poor reliability of sleep quality indices derived from a single day of monitoring. Wake after sleep onset was the most reliable with an estimate of ICC $=0.64$ (95\% CI: 0.59, 0.70). Sleep latency showed the least agreement with ICC $=0.33(95 \%$ CI: $0.27,0.39$ ). Reliability estimates for 7 days showed moderate-to-high reliability across all five sleep quality indices. Sleep duration had a reliability ICC $=0.84$ (95\% CI: $0.80,0.88)$ at 7 days, fragmentation index had an ICC $=0.90$ (95\% CI: 0.87, 0.92), sleep efficiency had an ICC $=0.92$ (95\% CI: 0.90, 0.94), sleep latency had an ICC $=0.73$ (95\% CI: 0.66, 0.79), and wake after sleep onset had an ICC $=0.93$ (95\% CI: 0.91, 0.94). Spearman-
Brown analyses indicated that in order to achieve reliability of ICC $=0.80$, sleep duration needed to be monitored for 5 days, fragmentation index for 4 days, sleep efficiency for 3 days, sleep latency for 12 days, and wake after sleep onset for 3 days.

\section{Older adults without $\mathrm{MCl}(\mathrm{N}=69)$}

There was poor reliability of sleep quality indices derived from a single day of monitoring for adults without MCI. Sleep efficiency was the most reliable with an estimate of ICC $=0.61$ (95\% CI: 0.53, 0.70), while sleep latency showed the least agreement with $\mathrm{ICC}=0.31(95 \% \mathrm{CI}$ : $0.23,0.41$ ). Reliability estimates for 7 days showed moderate-to-high reliability across all five sleep quality indices. Sleep duration had a reliability ICC $=0.86(95 \%$ CI: $0.81,0.91)$ at 7 days, fragmentation index had an ICC $=0.85$ (95\% CI: 0.79, 0.90), sleep efficiency had an ICC $=0.91 \quad$ (95\% CI: 0.87, 0.94), sleep latency had an ICC $=0.73$ (95\% CI: $0.62,0.82)$, and wake after sleep onset had an ICC $=0.91$ (95\% CI: $0.88,0.94)$. In order to

Table 2 Reliability coefficients

\begin{tabular}{|c|c|c|c|c|c|c|c|c|}
\hline & & \multicolumn{4}{|c|}{ Intraclass Reliability Coefficients (95\% CI) } & \multicolumn{3}{|c|}{$\begin{array}{l}\text { Days of Monitoring Required to Achieve Acceptable } \\
\text { Reliabilities of }\end{array}$} \\
\hline & & 1 Day & 4 Days & 7 Days & 14 Days & 0.7 & 0.8 & 0.9 \\
\hline \multirow[t]{5}{*}{$\begin{array}{l}\text { All Participants } \\
(N=151)\end{array}$} & Sleep Duration & $\begin{array}{l}0.46 \\
(0.40,0.52)\end{array}$ & $\begin{array}{l}0.71 \\
(0.63,0.78)\end{array}$ & $\begin{array}{l}0.84 \\
(0.80,0.88)\end{array}$ & $\begin{array}{l}0.92 \\
(0.89,0.93)\end{array}$ & 2.74 & 4.70 & 10.57 \\
\hline & Fragmentation Index & $\begin{array}{l}0.55 \\
(0.49,0.61)\end{array}$ & $\begin{array}{l}0.82 \\
(0.76,0.86)\end{array}$ & $\begin{array}{l}0.90 \\
(0.87,0.92)\end{array}$ & $\begin{array}{l}0.94 \\
(0.93,0.95)\end{array}$ & 1.91 & 3.27 & 7.36 \\
\hline & Sleep Efficiency & $\begin{array}{l}0.63 \\
(0.57,0.68)\end{array}$ & $\begin{array}{l}0.83 \\
(0.78,0.87)\end{array}$ & $\begin{array}{l}0.92 \\
(0.90,0.94)\end{array}$ & $\begin{array}{l}0.96 \\
(0.95,0.97)\end{array}$ & 1.37 & 2.35 & 5.29 \\
\hline & Sleep Latency & $\begin{array}{l}0.33 \\
(0.27,0.39)\end{array}$ & $\begin{array}{l}0.40 \\
(0.23,0.55)\end{array}$ & $\begin{array}{l}0.73 \\
(0.66,0.79)\end{array}$ & $\begin{array}{l}0.86 \\
(0.83,0.89)\end{array}$ & 4.74 & 8.12 & 18.27 \\
\hline & Wake After Sleep Onset & $\begin{array}{l}0.64 \\
(0.59,0.70)\end{array}$ & $\begin{array}{l}0.85 \\
(0.81,0.89)\end{array}$ & $\begin{array}{l}0.93 \\
(0.91,0.94)\end{array}$ & $\begin{array}{l}0.96 \\
(0.95,0.97)\end{array}$ & 1.31 & 2.25 & 5.06 \\
\hline \multirow{5}{*}{$\begin{array}{l}\text { Older Adults without Mild } \\
\text { Cognitive Impairment } \\
(N=69)\end{array}$} & Sleep Duration & $\begin{array}{l}0.52 \\
(0.43,0.62)\end{array}$ & $\begin{array}{l}0.75 \\
(0.64,0.84)\end{array}$ & $\begin{array}{l}0.86 \\
(0.81,0.91)\end{array}$ & $\begin{array}{l}0.93 \\
(0.91,0.95)\end{array}$ & 2.15 & 3.69 & 8.31 \\
\hline & Fragmentation Index & $\begin{array}{l}0.44 \\
(0.35,0.54)\end{array}$ & $\begin{array}{l}0.75 \\
(0.63,0.83)\end{array}$ & $\begin{array}{l}0.85 \\
(0.79,0.90)\end{array}$ & $\begin{array}{l}0.91 \\
(0.88,0.94)\end{array}$ & 2.97 & 5.09 & 11.45 \\
\hline & Sleep Efficiency & $\begin{array}{l}0.61 \\
(0.53,0.70)\end{array}$ & $\begin{array}{l}0.82 \\
(0.74,0.88)\end{array}$ & $\begin{array}{l}0.91 \\
(0.87,0.94)\end{array}$ & $\begin{array}{l}0.95 \\
(0.94,0.97)\end{array}$ & 1.49 & 2.56 & 5.75 \\
\hline & Sleep Latency & $\begin{array}{l}0.31 \\
(0.23,0.41)\end{array}$ & $\begin{array}{l}0.37 \\
(0.08,0.58)\end{array}$ & $\begin{array}{l}0.73 \\
(0.62,0.82)\end{array}$ & $\begin{array}{l}0.85 \\
(0.80,0.90)\end{array}$ & 5.19 & 8.90 & 20.03 \\
\hline & Wake After Sleep Onset & $\begin{array}{l}0.58 \\
(0.50,0.67)\end{array}$ & $\begin{array}{l}0.83 \\
(0.76,0.89)\end{array}$ & $\begin{array}{l}0.91 \\
(0.88,0.94)\end{array}$ & $\begin{array}{l}0.95 \\
(0.93,0.95)\end{array}$ & 1.69 & 2.90 & 6.52 \\
\hline \multirow{5}{*}{$\begin{array}{l}\text { Older Adults with Mild Cognitive } \\
\text { Impairment } \\
(N=82)\end{array}$} & Sleep Duration & $\begin{array}{l}0.40^{*} \\
(0.32,0.49)\end{array}$ & $\begin{array}{l}0.65^{*} \\
(0.51,0.76)\end{array}$ & $\begin{array}{l}0.80 \\
(0.73,0.86)\end{array}$ & $\begin{array}{l}0.90 \\
(0.86,0.93)\end{array}$ & 3.50 & 6.00 & 13.50 \\
\hline & Fragmentation Index & $\begin{array}{l}0.61^{* *} \\
(0.53,0.69)\end{array}$ & $\begin{array}{l}0.84^{*} \\
(0.78,0.89)\end{array}$ & $\begin{array}{l}0.91^{*} \\
(0.88,0.94)\end{array}$ & $\begin{array}{l}0.95^{* *} \\
(0.94,0.97)\end{array}$ & 1.49 & 2.56 & 5.75 \\
\hline & Sleep Efficiency & $\begin{array}{l}0.64 \\
(0.57,0.72)\end{array}$ & $\begin{array}{l}0.84 \\
(0.77,0.89)\end{array}$ & $\begin{array}{l}0.92 \\
(0.89,0.95)\end{array}$ & $\begin{array}{l}0.94 \\
(0.92,0.96)\end{array}$ & 1.31 & 2.25 & 5.06 \\
\hline & Sleep Latency & $\begin{array}{l}0.35 \\
(0.27,0.44)\end{array}$ & $\begin{array}{l}0.48 \\
(0.28,0.65)\end{array}$ & $\begin{array}{l}0.73 \\
(0.63,0.81)\end{array}$ & $\begin{array}{l}0.87 \\
(0.83,0.91)\end{array}$ & 4.33 & 7.43 & 16.71 \\
\hline & Wake After Sleep Onset & $\begin{array}{l}0.68^{*} \\
(0.61,0.75)\end{array}$ & $\begin{array}{l}0.86 \\
(0.81,0.91)\end{array}$ & $\begin{array}{l}0.93 \\
(0.91,0.95)\end{array}$ & $\begin{array}{l}0.97^{*} \\
(0.95,0.98)\end{array}$ & 1.10 & 1.88 & 4.24 \\
\hline
\end{tabular}


achieve reliability of ICC $=0.80$, sleep duration needed to be monitored for 4 days, fragmentation index needed to be monitored for 6 days, sleep efficiency needed 3 days, sleep latency needed 9 days, and wake after sleep onset needed 3 days.

\section{Older adults with probable $\mathrm{MCl}(\mathrm{N}=82)$}

We determined there was poor reliability of sleep quality from a single day of monitoring for all sleep quality indices in adults with probable MCI. Wake after sleep onset was the most reliable with an estimate of ICC $=0.68$ (95\% CI: $0.61,0.75)$, while sleep latency showed the least agreement with ICC $=0.35$ (95\% CI: 0.27, 0.44). Reliability estimates for 7 days showed modest-to-good reliability across all four sleep quality indices. Sleep duration had a reliability ICC $=0.80$ (95\% CI: $0.73,0.86)$ at 7 days, fragmentation index had an ICC $=0.91$ (95\% CI: $0.88,0.94$ ), sleep efficiency had an ICC $=0.92$ (95\% CI: 0.89, 0.95), sleep latency had an ICC $=0.73$ (95\% CI: 0.63, 0.81), and wake after sleep onset had an ICC $=0.93$ (95\% CI: 0.91, 0.95). Spearman-Brown prophecies indicated that in order to achieve reliability of ICC $=0.80$, sleep duration needed to be monitored for 6 days, fragmentation index and sleep efficiency needed 3 days, sleep latency needed 8 days, and wake after sleep onset needed 2 days.

\section{Differences in ICC estimates for different sleep parameters by cognitive status}

Significantly different ICCs are demarcated in Table 2. We determined that there were significantly different ICCs based on cognitive status for fragmentation index following 14 days $(z=2.34 ; p=0.02)$ and 1 day $(z=2.68$; $p<0.01)$ of monitoring. There were marginally different ICCs based on cognitive status for sleep duration following one $(z=1.85 ; p=0.06)$ and 4 days $(z=1.96 ; p=0.05)$ of monitoring, sleep fragmentation following four $(z=$ $1.89 ; p=0.06)$ and 7 days $(z=1.88 ; p=0.06)$, and wake after sleep onset following one $(z=1.78 ; p=0.08)$ and 14 days $(z=1.85 ; p=0.06)$.

\section{Discussion}

Our results indicate the MW8 provides reliable estimates of sleep after at least 7 days of observation for both older adults with MCI and those without. Specifically, we found that at least 7 days of monitoring-irrespective of cognitive status-provides good agreement for sleep duration $(\mathrm{ICC}=0.87$ ), fragmentation index (ICC $=0.87$ ), sleep efficiency (ICC $=0.91$, and wake after sleep onset $(I C C=0.93)$. Sleep latency provides moderate agreement after at least 7 days of wear $(\mathrm{ICC}=0.72)$. Given that four measures of sleep provide good reliability after 7 days, and one measure provides at least an acceptable level of agreement, we suggest the MW8 requires at least 7 days of consecutive wear to provide reliable estimates of sleep. Although our SpearmanBrown analyses indicate reliable estimates of fragmentation index, sleep efficiency, and wake after sleep onset can be achieved from less than 7 days of monitoring for both older adults with and without MCI, we also determined that at least 7 days of wear-time is needed to achieve a reliable estimate of sleep duration in older adults with MCI. Hence, we suggest that future investigations into the sleep of older adults with and without MCI should measure wrist-worn actigraphy for a period of at least 7 days.

Few studies have examined the number of nights needed to reliably estimate sleep. Acebo and colleagues determined the reliability of the Mini Motionlogger wrist-worn actigraph (Advanced Model, Ambulatory Monitoring Inc.) for measuring sleep in 169 young children (aged $1-5$ years) and 55 adolescents (11-16 years; (Acebo et al., 1999)). The results of this study indicated that five nights of monitoring was enough to provide good estimates of sleep in both young children and adolescents. A recent analysis of the MW8 in middle-aged men and women suggested that at least 7 days of observation was enough to provide reliable estimates of sleep (Aili et al., 2017), however we are only aware of two studies which have examined the reliability of sleep measures for older adults (Van Someren, 2007; Wohlgemuth et al., 1999). Wohlgemuth and colleagues determined that seven nights of monitoring using sleep logs and polysomnography was enough to reliably estimate sleep duration, sleep efficiency, and time in bed in both healthy older adults $(N=32)$ and older adults with primary insomnia $(N=32$; (Wohlgemuth et al., 1999)). More recently, Van Someren determined that seven nights of sleep monitoring using the Actiwatch (Cambridge Neurotechnology, Cambridge, UK), provides good estimates of reliability for sleep duration and sleep efficiency in both older adults with primary insomnia $(N=$ $10)$ and older adults with dementia $(N=12$; (Van Someren, 2007)). Our study of older adults with and without $\mathrm{MCI}$ appears to echo these results, and further indicates that estimates of sleep can be reliably measured using seven nights of actigraphy monitoring.

Our results also indicate that older adults with $\mathrm{MCI}$ have poorer sleep than their healthy cognitive peers. We determined that older adults with MCI have lower subjective and objective sleep duration than those without MCI after adjusting for age, sex, and sleep medication use. We also found that older adults with MCI appear to have more consistent fragmentation indices from nightto-night, but less consistent sleep duration from nightto-night. While these differences in ICCs do not warrant different monitoring protocols-given that 7 days of wear time provides good estimates of reliability for sleep duration and fragmentation index irrespective of 
cognitive status-it is interesting that older adults with MCI have different sleep patterns. One plausible explanation is that $\mathrm{MCI}$ associated changes in sleep quality include less regularity in sleep duration (with a tendency towards shorter sleep durations than cognitively healthy older adults) and consistently greater sleep fragmentation. This lower consistency in sleep duration from night-to-night appears to suggest that older adults with MCI might have less homeostatic sleep drive (i.e., Process S) than their healthy cognitive counterparts. It is also plausible that underlying changes in the brains of older adults with MCI - such as neural atrophy, nocturnal hypoxia, and altered neuromodulation - might alter their sleep (Scullin \& Bliwise, 2015). For example, older adults with $\mathrm{MCI}$ have greater amounts of $\mathrm{A} \beta$ accumulation (Pike et al., 2007), and greater $A \beta$ load is associated with more disrupted sleep (Mander et al., 2015). Future research should therefore examine why older adults with MCI experience different sleep patterns than their healthy cognitive counterparts, since these underlying differences could be related to their increased risk of dementia (Landry \& Liu-Ambrose, 2014).

While 7 days of wear-time provides good agreement for sleep duration, fragmentation index, and wake after sleep onset, this monitoring protocol only provides moderate agreement for sleep latency. However, sleep latency is difficult to accurately and reliably determine-even using polysomnography (Ancoli-Israel et al., 2003; Cantero et al., 2002). Actigraphic assessments of sleep latency are even more challenging since individuals with poor sleep tend to lie in bed motionless, but awake, for long periods of time (de Souza et al., 2003). Sleep latency may thus be difficult to accurately and reliably estimate using currently available field methods, and associations between sleep latency and cognitive health should be treated with caution.

\section{Limitations and future research}

Our study has only determined the reliability of MW8 in a sample of community-dwelling older adults. Use of this device in diseased populations may not yield accurate estimations of sleep quality after 7 days of measurement. While there is at least preliminary evidence of validity for the MW8 (O'Hare et al., 2015; Martoni et al., 2012; Elbaz et al., 2012), there is not yet criterion evidence of validity for the MW8 in older adults. Future research is thus needed to determine the validity of the MW8 for estimating sleep in older adults with and without MCI.

MCI is typically diagnosed using the Petersen criteria: 1) subjective cognitive impairment; 2) objective cognitive impairment according to a MoCA score of $<26 / 30$; 3 ) no functional impairment in activities of daily living; and 4) no dementia (Petersen, 2004). However, we did not use a clinical diagnosis of $\mathrm{MCI}$ in our study, but rather classified individuals as MCI solely based on a MoCA score of $<26 / 30$. All participants were likely free of dementia as they all had an MMSE score of $>24 / 30$ (Folstein et al., 1975).

We did not include napping in our calculations for each sleep variable, and thus our estimates of different sleep indices are exclusive to the major sleep period. Information outside of the major sleep period can be clinically informative, however sleep scoring algorithims for nighttime sleep estimation are not as well validated for scoring of daytime sleeping and naps (Ancoli-Israel et al., 2015). Thus, future research is needed to examine the reliability of indices of each total 24-h period of sleep for both older adults with and without MCI.

There are also significant changes which occur in sleep schedules, sleep architecture, and other aspects of sleep quality from middle-age to old-age (Crowley, 2011; Espiritu, 2008; Scullin \& Bliwise, 2015), and it is possible that the younger-older adults of our sample may have significant differences in sleep-wake patterns compared with the older-older adults. Our results should be applied with caution to older adults with significant sleep disturbances as well as older adults with chronic conditions, as these individuals may require different observation periods than our sample. Although we confirmed event-marker time stamps with the CSD to determine when a participant was awake and out of bed according to current actigraphy guidelines (Ancoli-Israel et al., 2015), it is possible that the last awakening during each sleep window did not coincide with the time participants got out of bed. Future research is needed to establish more accurate field methods for determining when older adults are awake and out of bed.

Our analyses were limited to five markers of sleep quality that are estimated by the MW8 (i.e., sleep duration, fragmentation index, sleep efficiency, sleep latency, and wake after sleep onset). We cannot provide guidance for other potential markers of sleep quality that may be estimated by the MW8. Other actigraphs will likely require different observation periods in order to collect reliable estimates of sleep quality.

\section{Conclusions}

In summary, the results of this study indicate that at least 7 days of MW8 wear-time is appropriate for populationbased field studies involving older adults. We determined that the MW8, an actigraphy device commonly used to measure physical activity (Landry et al., 2015b; Falck et al., 2017), provides reliable estimates of sleep for older adults with and without $\mathrm{MCI}$ after at least 7 days of observation. While there are differences in sleep quality based on cognitive status, similar measurement protocols can be used for older adults with MCI and those without MCI. These results provide researchers with a reliable tool to examine 
differences in sleep (as well as physical activity) in older adults with and without cognitive impairment, which may have future impact on the prevention of cognitive decline.

\section{Supplementary information}

Supplementary information accompanies this paper at https://doi.org/10. 1186/s41606-020-00048-w.

Additional file 1: Supplementary Material S1. $R$ statistical code. Additional file 2: Supplementary Material S2. Sensitivity Analyses when including participants without Montreal Cognitive Assessment scores.

\section{Abbreviations}

Aß: Amyloid-beta; ANCOVA: Analysis of Covariance; BMl: Body Mass Index; Cl: Confidence Interval; CSD: Consensus Sleep Diary; ICC: Intraclass Correlation; ICC 1 : Single Absolute Intraclass Correlation; ICC 2 k: Average Random Raters Intraclass Correlation; $\mathrm{MCl}$ : Mild Cognitive Impairment; MMSE: Mini-Mental State Exam; MoCA: Montreal Cognitive Assessment; MW8: MotionWatch8@ wrist-worn actigraph; PSQI: Pittsburgh Sleep Quality Index

\section{Acknowledgements}

We would like to thank the members of the Aging, Mobility, and Cognitive Neuroscience Laboratory for their assistance with recruitment and data collection.

\section{Authors' contributions}

RSF and TLA conceived the study concept and design. RSF wrote the first draft of the manuscript, performed all data analyses, and interpreted the results. PCYC was responsible for data collection and management. CKB, PCYC, and TLA each wrote portions of the manuscript and provided critical review. All authors approved of the final manuscript.

\section{Funding}

CKB is funded by Brain Canada and the Alzheimer's Association. Funding for this work was provided to TL-A by the Jack Brown and Family Alzheimer Research Foundation.

\section{Availability of data and materials}

The datasets used and analyzed during the current study are available from the corresponding author on reasonable request.

\section{Ethics approval and consent to participate}

Ethical approval for this study was obtained from the Vancouver Coastal Health Research Institute and the University of British Columbia's Clinical Research Ethics Board (H14-01301). All participants provided written informed consent.

\section{Consent for publication}

Not applicable.

\section{Competing interests}

The authors declare that they have no competing interests.

\section{Author details}

${ }^{1}$ Aging, Mobility and Cognitive Neuroscience Laboratory, Department of Physical Therapy, Faculty of Medicine, University of British Columbia, 212-2177 Wesbrook Mall, Vancouver, BC V6T 1Z3, Canada. ²Djavad Mowafaghian Centre for Brain Health, University of British Columbia, Vancouver, BC, Canada. ${ }^{3}$ Centre for Hip Health and Mobility, University of British Columbia, Vancouver, BC, Canada.
Received: 9 January 2020 Accepted: 6 June 2020

Published online: 16 June 2020

\section{References}

Acebo C, Sadeh A, Seifer R, Tzischinsky O, Wolfson AR, Hafer A, et al. Estimating sleep patterns with activity monitoring in children and adolescents: how many nights are necessary for reliable measures? Sleep. 1999;22(1):95-103.

Aili K, Åström-Paulsson S, Stoetzer U, Svartengren M, Hillert L. Reliability of actigraphy and subjective sleep measurements in adults: the design of sleep assessments. J Clin Sleep Med. 2017;13(01):39-47.

Ancoli-Israel S, Cole R, Alessi C, Chambers M, Moorcroft W, Pollak CP. The role of actigraphy in the study of sleep and circadian rhythms. Sleep. 2003;26(3): 342-92.

Ancoli-Israel S, Martin JL, Blackwell T, Buenaver L, Liu L, Meltzer LJ, et al. The SBSM guide to actigraphy monitoring: clinical and research applications. Behav Sleep Med. 2015;13(sup1):S4-S38.

Banks S, Dinges DF. Behavioral and physiological consequences of sleep restriction. J Clin Sleep Med. 2007;3(5):519.

Borbély AA, Daan S, Wirz-Justice A, Deboer T. The two-process model of sleep regulation: a reappraisal. J Sleep Res. 2016;25(2):131-43.

Buysse DJ, Reynolds CF, Monk TH, Berman SR, Kupfer DJ. The Pittsburgh sleep quality index: a new instrument for psychiatric practice and research. Psychiatry Res. 1989;28(2):193-213.

Cantero JL, Atienza M, Stickgold R, Hobson JA. Nightcap: a reliable system for determining sleep onset latency. Sleep. 2002;25(2):238-45.

Carney CE, Buysse DJ, Ancoli-Israel S, Edinger JD, Krystal AD, Lichstein KL, et al. The consensus sleep diary: standardizing prospective sleep self-monitoring. Sleep. 2012;35(2):287-302.

Crowley K. Sleep and sleep disorders in older adults. Neuropsychol Rev. 2011; 21(1):41-53.

da Silva RAPC. Sleep disturbances and mild cognitive impairment: a review. Sleep Sci. 2015;8(1):36-41

de Souza L, Benedito-Silva AA, Pires MLN, Poyares D, Tufik S, Calil HM. Further validation of actigraphy for sleep studies. Sleep. 2003;26(1):81-5.

Denegar CR, Ball DW. Assessing reliability and precision of measurement: an introduction to intraclass correlation and standard error of measurement. J Sport Rehabil. 1993:2(1):35-42.

Elbaz M, Yauy K, Metlaine A, Martoni M, Leger D. Validation of a new actigraph motion watch versus polysomnography on 70 healthy and suspected sleepdisordered subjects. J Sleep Res. 2012;21:218.

Espiritu JRD. Aging-related sleep changes. Clin Geriatr Med. 2008;24(1):1-14

Falck RS, Best JR, Davis JC, Liu-Ambrose T. The independent associations of physical activity and sleep with cognitive function in older adults. J Alzheimers Dis. 2018:63:1-16.

Falck RS, Landry GJ, Best JR, Davis JC, Chiu BK, Liu-Ambrose T. Cross-sectional relationships of physical activity and sedentary behavior with cognitive function in older adults with probable mild cognitive impairment. Phys Ther. 2017:97:975-84.

Foley DJ, Monjan AA, Brown SL, Simonsick EM, Wallace RB, Blazer DG. Sleep complaints among elderly persons: an epidemiologic study of three communities. Sleep. 1995;18(6):425-32.

Folstein MF, Folstein SE, McHugh PR. "Mini-mental state". A practical method for grading the cognitive state of patients for the clinician. J Psychiatr Res. 1975; 12(3):189-98.

Garrouste-Orgeas M, Troché G, Azoulay E, Caubel A, de Lassence A, Cheval C, et al. Body mass index. Intensive Care Med. 2004;30(3):437-43.

Hopkins KD. Educational and psychological measurement and evaluation: ERIC; 1998.

Jacamin-Gadda H, Alperovitch A, Montlahuc C, Commenges D, Leffondre K, Dufouil C, et al. 20-year prevalence projections for dementia and impact of preventive policy about risk factors. Eur J Epidemiol. 2013;28(6):493-502.

Ju Y-ES, Lucey BP, Holtzman DM. Sleep and Alzheimer disease pathology-a bidirectional relationship; 2013.

Kang J-E, Lim MM, Bateman RJ, Lee JJ, Smyth LP, Cirrito JR, et al. Amyloid- $\beta$ dynamics are regulated by orexin and the sleep-wake cycle. Science. 2009; 326(5955):1005-7.

Killgore WD. Effects of sleep deprivation on cognition. Prog Brain Res. 2010;185: 105-29 Elsevier.

Koo TK, Li MY. A guideline of selecting and reporting intraclass correlation coefficients for reliability research. J Chiropr Med. 2016;15(2):155-63.

Krystal AD, Edinger JD. Measuring sleep quality. Sleep Med. 2008;9:S10-S7. 
Landry GJ, Best JR, Liu-Ambrose T. Measuring sleep quality in older adults: a comparison using subjective and objective methods. Front Aging Neurosci. 2015a;7:166.

Landry GJ, Falck RS, Beets MW, Liu-Ambrose T. Measuring physical activity in older adults: calibrating cut-points for the MotionWatch 8๑. Front Aging Neurosci. 2015b;7:165.

Landry GJ, Liu-Ambrose T. Buying time: a rationale for examining the use of circadian rhythm and sleep interventions to delay progression of mild cognitive impairment to Alzheimer's disease. Front Aging Neurosci. 2014;6:325.

Langa KM, Levine DA. The diagnosis and management of mild cognitive impairment: a clinical review. JAMA. 2014;312(23):2551-61.

Liu-Ambrose T, Falck RS. Sleep, physical activity, and cognitive health in older adults. Handbook Behav Neurosci Elsevier. 2019;30:665-76.

Lowe CJ, Safati A, Hall PA. The neurocognitive consequences of sleep restriction: a meta-analytic review. Neurosci Biobehav Rev. 2017:80:586-604

Mander BA, Marks SM, Vogel JW, Rao V, Lu B, Saletin JM, et al. $\beta$-Amyloid disrupts human NREM slow waves and related hippocampus-dependent memory consolidation. Nat Neurosci. 2015;18(7):1051.

Martoni M, Bayon V, Elbaz M, Léger D. Using actigraphy versus polysomnography in the clinical assessment of chronic insomnia (retrospective analysis of 27 patients). Presse Med. 2012;41(3):e95-e100.

Naismith SL, Hickie IB, Terpening Z, Rajaratnam SW, Hodges JR, Bolitho S, et al. Circadian misalignment and sleep disruption in mild cognitive impairment. J Alzheimers Dis. 2014;38(4):857-66.

Nasreddine ZS, Phillips NA, Bédirian V, Charbonneau S, Whitehead V, Collin I, et al. The Montreal cognitive assessment, MoCA: a brief screening tool for mild cognitive impairment. J Am Geriatr Soc. 2005;53(4):695-9.

O'Hare E, Flanagan D, Penzel T, Garcia C, Frohberg D, Heneghan C. A comparison of radio-frequency biomotion sensors and actigraphy versus polysomnography for the assessment of sleep in normal subjects. Sleep Breathing. 2015;19(1):91-8.

Petersen R, Stevens J, Ganguli M, Tangalos E, Cummings J, DeKosky S. Practice parameter: early detection of dementia: mild cognitive impairment (an evidence-based review) report of the quality standards Subcommittee of the American Academy of neurology. Neurology. 2001;56(9):1133-42.

Petersen RC. Mild cognitive impairment as a diagnostic entity. J Intern Med. 2004;256(3):183-94

Petersen RC, Smith GE, Waring SC, Ivnik RJ, Tangalos EG, Kokmen E. Mild cognitive impairment: clinical characterization and outcome. Arch Neurol. 1999;56(3):303-8

Pike KE, Savage G, Villemagne VL, Ng S, Moss SA, Maruff P, et al. $\beta$-Amyloid imaging and memory in non-demented individuals: evidence for preclinical Alzheimer's disease. Brain. 2007;130(11):2837-44.

Roh JH, Huang Y, Bero AW, Kasten T, Stewart FR, Bateman RJ, et al. Disruption of the sleep-wake cycle and diurnal fluctuation of $\beta$-amyloid in mice with Alzheimer's disease pathology. Sci Transl Med. 2012;4(150):150ra22.

Sadeh $\mathrm{A}$. The role and validity of actigraphy in sleep medicine: an update. Sleep Med Rev. 2011;15(4):259-67.

Scullin MK. Do older adults need sleep? A review of neuroimaging, sleep, and aging studies. Curr Sleep Med Rep. 2017;3(3):204-14.

Scullin MK, Bliwise DL. Sleep, cognition, and normal aging: integrating a half century of multidisciplinary research. Perspect Psychol Sci. 2015;10(1):97-137.

Tranah GJ, Blackwell T, Stone KL, Ancoli-Israel S, Paudel ML, Ensrud KE, et al. Circadian activity rhythms and risk of incident dementia and mild cognitive impairment in older women. Ann Neurol. 2011;70(5):722-32.

Van Someren EJ. Improving actigraphic sleep estimates in insomnia and dementia: how many nights? J Sleep Res. 2007;16(3):269-75.

Wohlgemuth WK, Edinger JD, Fins Al, Sullivan RJ. How many nights are enough? The short-term stability of sleep parameters in elderly insomniacs and normal sleepers. Psychophysiology. 1999;36(2):233-44.

Xie L, Kang H, Xu Q, Chen MJ, Liao Y, Thiyagarajan M, et al. Sleep drives metabolite clearance from the adult brain. Science. 2013;342(6156):373-7.

Yaffe K, Falvey CM, Hoang T. Connections between sleep and cognition in older adults. Lancet Neurol. 2014;13(10):1017-28.

Zinkhan M, Berger K, Hense S, Nagel M, Obst A, Koch B, et al. Agreement of different methods for assessing sleep characteristics: a comparison of two actigraphs, wrist and hip placement, and self-report with polysomnography. Sleep Med. 2014;15(9):1107-14.

\section{Publisher's Note}

Springer Nature remains neutral with regard to jurisdictional claims in published maps and institutional affiliations.

\section{Ready to submit your research? Choose BMC and benefit from}

- fast, convenient online submission

- thorough peer review by experienced researchers in your field

- rapid publication on acceptance

- support for research data, including large and complex data types

- gold Open Access which fosters wider collaboration and increased citations

- maximum visibility for your research: over $100 \mathrm{M}$ website views per year

At BMC, research is always in progress.

Learn more biomedcentral.com/submissions 